\title{
Narrativa
}

\section{L'arrivo del pop. Evoluzioni tematiche e formali nel romanzo contemporaneo}

\section{Carlo Tirinanzi de Medici}

\section{(2) OpenEdition}

\section{Journals}

Edizione digitale

URL: https://journals.openedition.org/narrativa/362

DOI: 10.4000/narrativa.362

ISSN: 2804-1224

\section{Editore}

Presses universitaires de Paris Nanterre

\section{Edizione cartacea}

Data di pubblicazione: 1 décembre 2019

Paginazione: 115-125

ISBN: 978-2-84016-350-3

ISSN: $1166-3243$

Notizia bibliografica digitale

Carlo Tirinanzi de Medici, «L'arrivo del pop. Evoluzioni tematiche e formali nel romanzo

contemporaneo», Narrativa [Online], 41 | 2019, online dal 01 novembre 2021, consultato il 08 décembre 2021. URL: http://journals.openedition.org/narrativa/362 ; DOI: https://doi.org/10.4000/narrativa.362

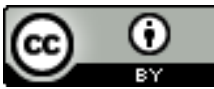

Narrativa est mise à disposition selon les termes de la Licence Creative Commons Attribution 4.0 International. 


\title{
L'arrivo del pop. Evoluzioni tematiche e formali nel romanzo contemporaneo
}

\begin{abstract}
RIASSUNTO
Il saggio traccia la traiettoria d'ingresso nella narrativa italiana di forme artistiche ed espressive derivate dalla cultura pop, verificando le fasi dell'assorbimento di nuovi media all'interno della forma romanzo. Dopo una parte introduttiva in cui si definisce il "pop" e si seguono le prime fasi di questo assorbimento, il saggio si concentra sulla narrativa degli anni Duemila, indagando alcuni usi specifici dell'immaginario pop attraverso i casi di Emanuela Carbé, Roberto Saviano, Walter Siti e Luca Rastello.
\end{abstract}

RÉSUMÉ

Cet article analyse la présence dans la prose narrative italienne des années 2000 de formes artistiques et expressives dérivées de la culture pop, en vérifiant les phases de l'assimilation de nouveaux médias à l'intérieur de la forme romanesque. Après avoir défini le "pop", et après avoir décrit les étapes de cette assimilation, cet article se concentre sur quelques œuvres des années 2000 pour enquêter sur le rôle de l'imaginaire pop chez des auteurs comme Emanuela Carbé, Roberto Saviano, Walter Siti et Luca Rastello.

1. Questo articolo vuole seguire le forme del pop quando penetrano nell'immaginario romanzesco, prima a livello tematico, poi (a partire dagli anni Novanta) a livello formale. Non è facile definire "pop". Il termine ovviamente è l'abbreviazione di "popular", ma cosa sia "popolare" è oggetto di lungo dibattito $^{1}$. Approfittando del diritto alla semplificazione concesso a interventi come questo, intendo per "cultura pop" la popular culture ai tempi della comunicazione

1. Sulle varie definizioni di "popular" che si sono succedute nel corso del tempo, riferite a diversi periodi storici, v. STORey John, Inventing Popular Culture, Malden, Blackwell, 2003. 
di massa ${ }^{2}$. L'interazione tra arte highbrow e popolare c'è sempre stata, e il Novecento non fa eccezione: dalla Neovanguardia alla pop art a certi esperimenti di Calvino (Cosmicomiche, Castello), la cultura di massa è parte integrante dell'orizzonte estetico moderno.

Ma a un certo punto muta la modalità di questa interazione. Fino agli anni Sessanta il processo di contaminazione è avvenuto secondo una strategia topdown: attori riconosciuti del campo culturale hanno assorbito modalità e forme espressive pop inglobandole nel proprio regime estetico. Con la generazione degli anni Cinquanta (quella nata nell'era di televisione, musica "leggera", film, ecc.) il processo è diventato bottom-up. La cultura $p o p$ non è stata più qualcosa di estraneo che si inseriva in un sistema preesistente, ma gli autori la riconoscevano come parte del proprio orizzonte culturale e, anche, della loro memoria estetica.

In Italia questo fenomeno nasce nella scrittura giovanile degli anni Settanta (Rocco e Antonia, i "Franchi narratori" di Feltrinelli, Enrico Palandri) ${ }^{3}$ ed esplode con Pier Vittorio Tondelli. Sin dal suo esordio Altri libertini (1980) i riferimenti al $p o p$ diventano organici all'opera. Diverse sue opere prendono a riferimento gli oggetti culturali di massa che lui e il suo pubblico conoscono bene. Racconta di videogame, accompagna Rimini con una "colonna sonora", usa molto la musica leggera; sfruttando i riferimenti pop come "segni generazionali"'. Un processo comune ad altri narratori coevi: si veda, ad esempio, Andrea De Carlo, dal sogno americano derivato dai film, sfondo di Treno dipanna (1981), alla televisione centrale in Macno (1984) e Yucatan (1986), allimmaginario legato a TV e fumetti dei suoi protagonisti ${ }^{5}$.

2. Così Dwight MacDonald e Ernest van den Haag. Più recente è la posizione di Schils Edward, "Mass society and its culture", in ID., Davison Peter, MeYersohn Rolf, Literary Taste, Culture, and Mass Communication, I, Cambridge, Chadwyck Healey, 1978, p. 35 ss.; Friske John, Understanding Popular Culture, London, Routledge, 2001. La centralità dei mass media è carattere proprio del $p o p$ secondo STRINATI, Dominic, $A n$ Introduction to Theories of Popular Cultures, 2nd ed., London, Routledge, 2005, p. 22 ss.

3. Si ricordi inoltre che l'esordio di Antonio Tabucchi con Piazza d'Italia riprende moduli cinematografici: cfr. Rimini Thea, "La cine(biblio)teca di Tabucchi", in Italies, 2007, pp. 321-348.

4. Cfr. Mondello Elisabetta, In principio fu tondelli, Milano, Il Saggiatore, 2007, pp. 26, 84 e passim.

5. Manai Franco, "Feinte séduction et autoréférentialité: les romans d'Andrea De Carlo", in Cahiers d'études italiennes 2008, pp. 103-113: p. 110. Cfr. "Intervista ad Andrea De Carlo", a cura di G. De Luca, in Italica, n. 84, 2007, pp. 838-842. 
Di lì in avanti gli autori mettono in scena il "melting pot culturale" in cui sono cresciuti: fenomeno evidentissimo nelle antologie Under 25 curate proprio da Tondelli. Interessante è il terzo volume della serie, Papergang: in esso ha un ruolo centralissimo la televisione, che nel decennio seguente (primo esempio La guerra degli Antò di Silvia Ballestra) dilagherà, forse anche in virtù della "discesa in campo" del proprietario di Mediaset Silvio Berlusconi. Negli anni Novanta "tutto entra nel letterario": oggi si riconosce in movimenti diversi (giovane narrativa, cannibali, pulp, noir...) una medesima tendenza a utilizzare il formulario dei mass media. Sono opere in cui la transcodifica assurge a strumento primario della composizione. In passato il romanzo ha assorbito aspetti di altre $\operatorname{arti}^{8}$; adesso accade lo stesso con le forme massmediatiche ${ }^{9}$, così che si intersecano elementi compositi e precari, pescati in un caotico magazzino che comprende letteratura di genere e classici, canzonette, videogiochi e videoclip, fumetti, programmi televisivi, spot pubblicitari, cronaca nera, saggi eruditi ecc. ${ }^{10}$

Questo bumus sostituisce la memoria propriamente letteraria nelle nuove generazioni ${ }^{11}$, ancora viva nel decennio precedente ${ }^{12}$.

A questa altezza storica si stabilizza anche in Italia un nuovo utilizzo del pop, non solo tematico o identitario, ma espressivo. Televisione, cinema $^{13}$, musica ${ }^{14}$

6. Galato Franco, “Cercatori di storie, videostorie e controstorie. Dieci percorsi di lettura", in Galato Franco, Panzieri Fulvio, Cardone Raffaele, Altre storie, Milano, Marcos y Marcos, 1996, p. 89.

7. Mondello Elisabetta, In principio fu Tondelli, cit., p. 19.

8. Cfr. almeno Kundera Milan, L'arte del romanzo, Milano, Adelphi, 1986, e ID., I testamenti traditi, Milano, Adelphi, 1994.

9. Del resto è in corso un sommovimento nel campo artistico, ormai da alcuni decenni, che vede vieppiù includere nel concetto forme prima sanzionate come para-artistiche (neologismo coniato sul modello di "para letterario"): il fumetto a partire dagli anni Novanta, oggi è il turno delle serie televisive: cfr. Rossini Gianluigi, Le serie TV, Bologna, Il Mulino, 2016; PIgA Emanuela, Romanzi e serie TV, Pisa, Pacini, 2018.

10. Meneghelli Andrea, "Il cinema italiano e le nuove leve letterarie", in Annali d'italianistica, n. 17, 2009, pp. 203-16, p. 206.

11. SimonetTi Gianluigi, "I nuovi assetti della narrativa italiana", in Allegoria, n. 57, 2008, pp. 95-136: p. 98; tratto del romanzo italiano contemporaneo è l'“abbandono della tradizione letteraria come baricentro della cultura del romanzo". Cfr. LA PORTA Filippo, La nuova narrativa italiana. Travestimenti e stili di fine secolo, $2^{\mathrm{a}}$ ed., Torino, Bollati Boringhieri, 2002, p. 262.

12. Cfr. Tani Stefano, Il romanzo di ritorno, Milano, Mursia, 1990.

13. Cfr. Senardi Fulvio, "Cannibali in Mitteleuropa", in Problemi, n. 109, 1997, pp. 262-284; Carotenuto Aldo, Il fascino discreto dell'orrore, Bompiani, Milano, 2012, pp. 289 ss.

14. LUPERINI Romano, "Giovani e cannibali solo basic instinct", in L'Indice dei libri del mese, n. 3, 1997, p. 10. 
diventano fonti di tecniche narrative a vari livelli: titolazione (molti i romanzi che si riferiscono a opere musicali o cinematografiche $)^{15}$, architettura ${ }^{16}$, lessico e strutturazione del periodo. Il ritmo rapido ${ }^{17}$ e "la voglia di enfasi, di iperbole" 18 sono dovuti a TV e videoclip. Alcuni autori si spingono a teorizzare il melting pot: la "scrittura rock" di Enrico Brizzi o quella "televisiva" di Aldo Nove ${ }^{19}$.

Dal pop entrano poi le forme narrative "basse" o paraletterarie, fonti di ambientazioni e personaggi: la stessa antologia Gioventù cannibale doveva in origine essere un'antologia dell'horror, si diffonde una "scuola pulp", e fa il suo ingresso nella narrativa autoctona "seria" il noir. Non da meno è la fantascienza, che informa (con toni fumettistici, peraltro) Il mondo è parcheggiato in discesa (2002), ultima opera di uno dei grandi narratori del periodo, Matteo Galiazzo. Gli anni Novanta sono il momento in cui il pop non solo entra, ma sfonda: la cifra in comune alle narrazioni di questo periodo è un accumulo di materiali che raggiunge la saturazione, "non una fuga dai media ma un'immersione nei media"20.

15. Tra i molti esempi Ballestra Silvia, Compleanno dell'Iguana, Ancona, Transeuropa, 1991 ("Iguana" è il soprannome di Iggy Pop), Brizzi Enrico, Jack Frusciante è uscito dal gruppo, Ancona, Transeuropa, 1994 (riferimento al chitarrista dei Red Hot Chili Peppers), Lucarelli Carlo, Almost blue, Torino, Einaudi, 1997 (celebre brano di Chet Baker); Meacci Giordano, Il cingbiale che uccise Liberty Valance, Roma, Minimum Fax 2016 (che riprende il film di John Ford del 1962). Il fenomeno è parte di un movimento più ampio, sovranazionale (come sovranazionale è il campo romanzesco), che parte almeno dalla tolstojana Sonata a Kreutzer (1889) e che ha visto negli ultimi anni Houston Nancy, Les variations Goldberg, Paris, Seuil, 1981, Fuller Jack, The Best of Jackson Payne, Chicago, University of Chicago Press, 2000, Josipovici Gabriel, Goldberg: Variations, Manchester, Carcanet, 2002.

16. Fonderia Italghisa di Giuseppe Caliceti, per esempio, è diviso in due "tempi", come un film; Destroy di Isabella Santacroce è strutturato come un CD, a tracce; in L'ora preferita della sera di Valeria Viganò le canzoni sono spunto diretto per i racconti.

17. SITI Walter, "Il tempo veloce del romanzo contemporaneo", in CASADEI Alberto (a cura di), Spazi e confini del romanzo, Bologna, Pendragon, 2002, pp. 261-265; SiMONETTI Gianluigi, La letteratura circostante, Bologna, Il Mulino, 2018, pp. 36 ss. Sull'origine nell'area giovane e cannibale di questo carattere, cfr. Sinibaldi Marco, Pulp, Roma, Donzelli, 1997, pp. 20 ss.

18. SimonetTi Gianluigi, La letteratura circostante, cit., p. 45.

19. "La scrittura rock non è tanto una scrittura che ha come argomento il rock, ma qualcosa di simile a uno spirito, a un'intonazione", ha dichiarato Brizzi in GASPODINI Cristina, Il mondo secondo Frusciante Jack, Ancona, Transeuropa, 1999, p. 76. In un'intervista concessa da Nove, invece, si legge: "Il mio scopo dichiarato era appunto quello di riportare il ritmo dello zapping in letteratura, scrivere televisivamente, ciò che è breve, veloce, spezzato" (Bonadonna Claudia, "Now Generation. Aldo Nove", in Pulp, 2, 1996, pp. 17-18).

20. Mondello Elisabetta, "La giovane narrativa italiana degli anni Novanta", in EAD. (a cura di), La narrativa italiana degli anni Novanta, Roma, Meltemi, 2004, pp. 11-38, p. 15. 
2. Negli anni Zero le cose tendono a stabilizzarsi e cadono gli eccessi del decennio precedente, ma il fenomeno è entrato pienamente nel circuito letterario "alto". In una delle più importanti - e impegnate - antologie d'inizio millennio, La qualità dell'aria (2005), i racconti d'invenzione tendono perlopiù al pop. Da una satira che riprende esplicitamente i moduli cannibali ${ }^{21}$ a racconti che spingono sulla narrativa di genere, spesso fantastici o con tendenze fantasmatiche (Covacich, nel racconto del quale la concretezza della città svanisce subito in sogno; Pugno, che anticipa i temi cyberpunk di Sirene, Pincio, il quale costruisce tutto il racconto sulla dicotomia reale/inventato). C'è anche un racconto grafico, Il maiale.

E la dimensione grafica è una delle caratteristiche di Mio salmone domestico (2013), romanzo d'esordio di Emanuela Carbé, esempio lampante della mutazione del serbatoio formale cui si ispirano i narratori degli anni Zero. Si tratta del racconto autodiegetico delle vite quotidiane (problemi sentimentali, esami da dare, uscite serali ecc.), raccontate con un piglio al limite del fiabesco, della protagonista, studentessa universitaria fuori sede, e del suo coinquilino: Crodo, un salmone parlante dal carattere tutt'altro che facile. Intorno ai due si muove una selva di personaggi fortemente stilizzati: compagni di università, il fidanzato Coso, amici di amici.

Lo stile del libro - frammentato, che procede per singulti e strappi - non è tanto un richiamo a esperienze sperimentali, quanto l'effetto diretto del luogo della gestazione di Mio salmone: un blog, "Lumicino", gestito da Carbé dal 2005. Un tratto comune ad altre opere nate nei tardi anni Zero, quando i blog hanno rivoluzionato la comunicazione (anche quella letteraria): Il mondo deve sapere (2006), ad esempio, di Michela Murgia. Ma in Carbé la tecnica diventa sguardo, informa il mondo percettivo della protagonista ("io per me funziono a brevi tratti, a segmenti, a pezzi") 22 . Il "discontinuo" calviniano ${ }^{23}$ (e Calvino presiede Mio salmone) domina la scena, senza che produca le ubbie suscitate in un Qfwfq o il senso di terribile esaltazione dei romanzi postmodernisti (a partire da Rumore

21. Nel racconto di Pacifico i protagonisti comprano casa via ebay; in "Un muro di televisori" l'11 settembre 2001 è vissuto in un centro commerciale.

22. Carbé Emanuela, Mio salmone domestico, Roma-Bari, Laterza, 2013, p. 136.

23. Vasta, e ancora bisognosa di studio, la riflessione di Calvino sul discontinuo, sul frammentario, il separato come condizione del pensiero contemporaneo - così si esprime in "Cibernetica e fantasmi", ora in CALvino Italo, Una pietra sopra, Torino, Einaudi, 1980 -, parte integrante della sua pratica e riflessione letteraria: dal racconto "Mitosi" contenuto in Ti con zero (1967) fino all'incompiuta lezione americana sulla consistency, per non parlare della forma appunto discontinua, fatta di calcolatissimi assemblaggi, che assumono spesso i suoi libri. 
bianco). La commistione di reale e meraviglioso avvicina il libro a certi cartoni animati giapponesi (Crodo è la versione incazzereccia di Spank), mentre la posizione della protagonista, come indifesa nei confronti di una vita che ancora non sa gestire (e Crodo è un filtro tra lei e il mondo, una membrana che attutisce i colpi, che fornisce una prima interpretazione di quanto accade $)^{24}$ richiama il tema del coming of age. Carbé (ventiduenne quando inizia a lavorare a Mio salmone) insomma scrive un racconto postadolescenziale adottando strategie postadolescenziali radicate nella sua generazione (manga, cartoni animati). L'ultima trentina di pagine del libro sono un racconto a fumetti, segno della spontaneità del passaggio da un medium all'altro.

3. L'interazione tra media diversi non è nuova (il Modernismo utilizza di frequente sottotesti musicali, ad esempio, e si veda l'opera di Milan Kundera) ${ }^{25}$, e in particolare le arti visive hanno spesso influenzato la costruzione romanzesca ${ }^{26}$. Ma la rimediazione è caratteristica propria soprattutto dei mass media ${ }^{27}$ : il pluralismo mediale, oggi, non è arma analogica per ovviare alla mancanza di senso del mondo, ma strategia per rappresentarlo, come ha dimostrato Carlo Lucarelli, a suo agio nel passaggio da un medium all'altro, adattando le strategie televisive alla pagina e viceversa (i costanti riferimenti alla struttura da romanzo giallo nella serie TV Blu notte, l'inserimento ecfrastico di immagini e filmati nei suoi romanzi). Ma la forma transmediale influenza anche l'ideologia del testo. Gomorra (2006), considerato segno del "ritorno al reale" della narrativa italiana ${ }^{28}$, deve molto alle tecniche massmediatiche, dalle quali deriva la sua "attitudine pop"29, Miconi vi

24. Anche le sagome di cartone che popolano il libro sono una riproduzione incapace di far male di quanto c'è all'esterno. Cfr. CorTellessa Andrea, recensione a Mio salmone domestico, Nazione indiana, 3 agosto 2013, https://www.nazioneindiana. com/2013/08/03/emmanuela-carbe-mio-salmone-domestico/

25. Cfr. Rizzante Massimo, "Fuga romanzesca. Note su La lentezza e L'identità di Milan Kundera", Nazione indiana 21 gennaio 2015, https://www.nazioneindiana. com/2015/01/21/la-fuga-romanzesca-note-su-la-lentezza-e-lidentita-di-milan-kundera/; Petermann Emily, The musical novel, Rochester, Camden House, 2014.

26. Cfr. Brook Peter, Lo sguardo realista, Roma, Carocci, 2017; Brogi Daniela, Un romanzo per gli occhi, Roma, Carocci, 2018.

27. Bolter Jay David, Grusin Richard, Remediation, Milano, Guerini, 2002.

28. Spinazzola Vittorio, La riscoperta dell'Italia, in Tirature '10, Milano, Il Saggiatore, pp. 10-15, ma si veda il ruolo nell'idea di "ritorno alla realtà" su cui verteva il celebre numero di Allegoria (57, 2008; cfr. Donnarumma Raffaele, Ipermodernità, Bologna, Il Mulino, 2014).

29. L'espressione viene dal discusso saggio di Wu Ming 1 sul New Italian Epic (Torino, Einaudi, 2009), il quale - al di là del giudizio complessivo - ha il merito di individuare le contiguità tra romanzo e $p o p$. 
vede "quel doppio livello tra ripetitività delle formule e allargamento dei territori

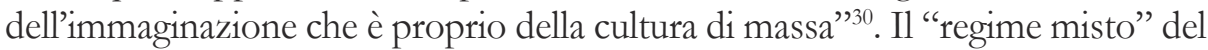
romanzo ${ }^{31}$ si deve all'influenza delle scritture d'informazione (inchiesta, memoir ecc.) ma centrali per l'opera sono anche televisione e cinema.

Gomorra è infarcito di citazioni cinematografiche, perlopiù inerenti ai film di mafia (Il camorrista, Scarface, Quei bravi ragazæi, Donnie Brasco): essi informano le descrizioni del mondo ${ }^{32}$ e a volte diventano frame interpretativo. L'idea della criminalità organizzata come prolungamento del capitalismo è nel Padrino (leitmotiv dei film è "è solo businéss"), mentre il protagonista-eroe "puro" 33 ha molto in comune con i film più didascalici di questo genere (come Bronx), forse per reazione al blurring dei confini morali messo in scena da quelle opere, delle quali i criminali sono affascinanti protagonisti. E cinematografico - cult - è l'urlo finale del libro ("Maledetti bastardi, sono ancora vivo!") 34 , preso da Papillon ${ }^{35}$. Anche quando lo contesta, Saviano pone il cinematografico come orizzonte di senso ${ }^{36}$.

Meno evidente, ma non meno pervasivo, è il ruolo della televisione, alla quale si deve il gusto esotista per il turismo dell'orrore ${ }^{37}$ e il nesso moralismo-emotività che informa tutte le sue pagine. Quando racconta, Saviano non riesce a separare il racconto da sé stesso. Ciò implica che tutto è filtrato dalla sua soggettività, specie dalle sue emozioni ${ }^{38}$. Nemmeno il paesaggio è

30. Giovannetti Paolo e Miconi Andrea, “Confondendo un po’ le carte (e i bit) in tavola", in L'Ulisse, n. 19, 2009, p. 8.

31. Donnarumma Raffaele, Ipermodernità, cit., p. 191.

32. Sul cinema come modello per la rappresentazione di Saviano, v. Giglioli Daniele, "Come farebbe Auerbach? Realismo postmoderno e separazione degli stili", in Moderna, n. 1-2, 2009, pp. 189-204: p. 196.

33. Luperini Romano, "Per Roberto Saviano", in Aetnanet, 21 gennaio 2009, http:// www.aetnanet.org $/$ modules.php?name $=$ News\&file $=$ article\&sid $=9762$

34. Saviano, Roberto, Gomorra, Milano, Mondadori, 2006, p. 331.

35. Weber Luigi, "Serpico, Scarface e Papillon", in Studi culturali, n. 3, 2007, pp. 523 534: p. 531; Giglioli Daniele, Come farebbe Auerbach?, cit., p. 198.

36. Così raccontando l'omicidio di Giuseppe e Romeo, i due camorristi wannabe uccisi perché hanno pestato i piedi a un clan: "Non chiamarono neanche anonimamente la polizia o un'ambulanza. Lasciarono che le mani dei cadaveri dei ragazzini fossero beccate dai gabbiani e le labbra e i nasi mangiucchiati dai randagi che circolavano sulle spiagge di spazzatura. Ma questo i film non lo raccontano, si fermano un attimo prima”. SAVIANO, Roberto, Gomorra, cit., p. 228.

37. SimonetTi Gianluigi, La letteratura circostante, cit., p. 114.

38. Cfr. Peldini Pierluigi, "Lo scrittore come intellettuale", in Allegoria, n. 63, 2012, pp. 135-163: p. 155. 
immune da questa distorsione ipersoggettiva ${ }^{39}$ che talvolta giunge a effetti discutibili, come nella scena del funerale di Annalisa Durante, con il telefono fatto squillare dalle amiche come forma di saluto. Scena che non solo è falsa (aprendo al problema dello scrupolo documentario in un libro che, per l'autore, è "tutto vero"), ma soffusa di "patetismo d'accatto" 40 . La struttura dell'opera, "dispersiva, accumulativa, policentrica [...] che non ha trama avvincente né eroi votati all'esemplarità" 41 , riprende quella di certi programmi-contenitore del pomeriggio, o dei talk show in prima serata ${ }^{42}$. Le storie individuali si susseguono davanti agli occhi dello spettatore senza lasciare traccia; perché siano significative il narratore le carica emotivamente.

La tendenza intermediale aumenta nelle opere successive: La paranza dei bambini, "è integralmente nell'osmosi di scrittura e media: è già un film o un serial, è davvero nutrito di cultura e subcultura mediatica, ed è davvero un romanzo" Lo stesso spostamento del piano dell'analisi, che dall'abbozzo economico-politico (omologia tra camorra e tardo capitalismo) di Gomorra va al moralistico-indignato (la criminalità, la droga "sono male", i malviventi e il fascino del male) di Zerozerozero e La paranza dei bambini sembra funzionale a una dimensione televisiva, in cui le sfumature non sono ammesse. Lo stesso si può dire del linguaggio, che tende al formulaico, allo stereotipato "di chiara matrice giornalistica" quando non "da rotocalco" 44

Ma proprio nell'idea della "parola come azione" - nell'interesse per la performance - che si sviluppa l'aspetto più legato ai moderni media di massa: Saviano è maestro nella "retorica del 'mettersi in gioco", che è "centrale in certe impostazioni critiche ma anche in molti altri ambiti dell'odierna società della comunicazione (è alla base per esempio delle logiche dei talent show)" ${ }^{\prime 4}$.

39. Saviano contamina «la rappresentazione dello spazio urbano con impressioni personali [...]. I suoi non sono mai paesaggi oggettivi, ma descrizioni in cui la linea delle case e delle strade si confonde con il discorso vissuto o col monologo interiore». BALDI Valentino, "Raccontare la città", in Allegoria, n. 69-70, 2015, pp. 61-74: p. 62.

40. Bertoni Clotilde, Letteratura e giornalismo, Roma, Carocci, 2009, p. 76.

41. Peldini Pierluigi, "Lo scrittore come intellettuale", cit., p. 151.

42. Fenomeno già presente in De Carlo quando assorbe nella sua prosa la televisione: cfr. Moestrup Jorn, "L'opera di Andrea De Carlo", in Atti del convegno internazionale "Rinnovamento del codice narrativo in Italia dal 1945 al 1992", vol. I, Roma, Bulzoni, 1995, pp. 649-59: p. 653.

43. Donnarumma Raffaele, recensione a R. Saviano, La paranza dei bambini, in Allegoria, n. 75, 2017.

44. Матт Luigi, Forme della narrativa italiana di oggi, Roma, Aracne, 2014, p. 249.

45. Ibid., p. 13. 
In quest'ottica rientra anche il recupero non del Pasolini storico, ma l'icona intellettuale pop, il "mito-Pasolini" di cui parla Walter Siti". Tutto in Saviano tende a un "sublime pop" o anche a una "catarsi pop", per cui alla cruda oggettività la tragedia conferisce un di più d'intensità, sommergendo lo spettatore di sentimenti lo ricatta moralmente per fargli accettare la sua versione della vicenda.

4. Un uso assai più consapevole delle strutture mediali è in Troppi paradisi (2006) di Walter Siti, un romanzo realista ai tempi del reality. Walter si espone esibizionisticamente sulla pagina come un "personaggio" televisivo (in esergo c'è una frase attribuita all'editor di Einaudi Ernesto Ferrero: "Faccia il mostro, e non rompa le scatole", e Walter non lesina particolari imbarazzanti), per cui il piano biografico del libro è funzionale al secondo, relativo al lavoro di Walter, autore di talk show. Entrambi sono sussunti dal terzo piano, saggistico (Walter interviene frequentemente per commentare, spiegare, dar conto di quanto accade sulla scena $)^{47}$ con salti netti di piano (gli scarti sono evidenziati da accorgimenti tipografici come parentesi o corsivi utilizzati per sottolineare i concetti; congiunzioni che segnalano un ragionamento come "quindi", "dunque", ecc.). Sembra il gesto di certi intellettuali da talk show, per i quali la tragedia (un infanticidio, un omicidio-suicidio, una strage) è il trampolino per discutere di massimi sistemi, emblemi della TV del dolore che giustifica il proprio voyeurismo attraverso discorsi "seri".

La televisione dà dunque forma al racconto: Walter è "come tutti" ed eccezionale, nella miglior tradizione della "TV-verità". Ma a fronte delle pretese di autenticità (nomi propri, luoghi reali, persino il pudore con cui Walter circonda la propria cerchia garantendone la privacy, come nel caso dell'amico pedofilo), alcune incongruenze quasi invisibili fanno sospettare che si tratti di una messinscena ${ }^{48}$ : il lettore si trova, per così dire, con un sassolino finzionale nella scarpa del realismo, ed è spinto a interrogare la pretesa di autenticità del testo, giungendo a individuarne la natura artificiosa. Con ciò, proprio mentre si imita il principio di funzionamento del reality, lo si enuncia ${ }^{49}$, in tal modo rompendo

46. Cfr. Siтt Walter, "Il mito Pasolini”, in Micromega, n. 6, 2005, pp. 135-139. Cfr. Tirinanzi De Medici Carlo, Il romanzo italiano contemporaneo, Roma, Carocci, 2018, pp. 201 203. Gianluigi SimONETTI parla di "pasolinismo di base": La letteratura circostante, cit., p. 370.

47. Un'analisi più dettagliata di questo fenomeno è in Tirinanzi De Medici Carlo, Il vero e il convenzionale, Torino, Utet, 2012, pp. 91-113.

48. Cfr. Marchese Lorenzo, L'io possibile, Massa, Transeuropa, 2014; 'Tirinanzi De Medici Carlo, "Su alcuni aspetti dell'autofinzione", in Il Verri, n. 63, 2017, pp. 22-44.

49. "Nel talk invece, e nel reality, la realtà-realtà fa valere tutti i propri diritti di interdizione e di inibizione: chiede ai protagonisti (o "ospiti", gente comunque in 
l'illusione di veridicità e rendendo visibile ciò che nella TV è occultato. Si smonta il meccanismo mentre lo si costruisce, perché se si aderisce alla realtà non la si può rappresentare; così il libro si costruisce intorno alle riflessioni di Walter (piano saggistico), unico strumento per superare la retorica della presa diretta e del reality: "Stare a una spanna di distanza dall'empiria è condizione necessaria per poterla esprimere compiutamente" ${ }^{50}$, e ciò spinge a uscire dal discorso televisivo-autofinzionale. Una cura omeopatica alla retorica dell'autenticità e alla logica dell'edizione straordinaria che dominano la nostra epoca.

Altrettanto scaltro è l'uso del pop in Piove all'insù di Luca Rastello (2006). Il romanzo si compone di 94 sottocapitoli, ognuno costituito da una mail che il protagonista, Pietro Miasco, manda alla sua compagna, licenziata in tronco, nell'attesa di raggiungerla. Coerentemente con la loro origine, ogni sottocapitolo ha un "oggetto". La natura iperfunzionale della mail, già utilizzata nei moderni romanzi epistolari ${ }^{51}$, si trasforma qui in altro. I titoli sono legati in modo "spesso ellittico e suggestivo" all'argomento, una controstoria del contemporaneo. "L'età di Tersite", come viene definita la nostra era - un'era basata sul rifiuto della responsabilità, sul gioco, sulla mancanza di serietà -, nasce dagli "esperimenti" dei giovani ribelli del Settantasette, che non hanno saputo controllare la trasformazione delle coscienze, così che invece dell" "uomo nuovo" preconizzato dal marxismo si ottiene un "homunculus". Ma Rastello - a differenza di altri, a partire da Houellebecq - non oppone alla nostra un'età dell'oro: non ne rigetta i suoi presupposti, piuttosto cerca di recuperare calvinianamente ciò che inferno non è, tornando alle sorgenti di quella cultura: l'utopia, espressa tramite la sua forma più avantpop, la fantascienza a sfondo sociale, che riassume e svela il piano metaforico del libro (ogni capitolo si chiude con il riassunto di uno di questi romanzi, mostrandone il legame con la nostra storia).

5. In un'epoca dominata dallo storytelling, che è stata definita "della narrazione infinita ${ }^{52 ",}$, il romanzo si fa carico dei caratteri narrativi di altri media, come ha

carne e ossa) di "essere come tutti" ma contemporaneamente di fare audience, cioè di incarnare l'eccezione, il mostro che il pubblico vuole vedere. Naturalmente nessuno ci sta a fare la parte del mostro [...]. I personaggi si sottraggono alla loro coerenza di personaggi: non vogliono sentire ragioni”, SIтI Walter, Troppi paradisi, Torino, Einaudi, 2006 , p. 354.

50. Ibid., p. 355.

51. A partire da Norman X e Monique Z, Norman e Monique, Torino, Einaudi, 1996, fino al recente Bondoux, Anne-Laure e Mourlevat Jean-Claude, Danzerò con te, Milano, Longanesi, 2016, ma Jonathan Coe ne ha sempre fatto largo uso.

52. Anselmi Gian Mario, L'approdo della letteratura, Roma, Carocci, 2018, p. 137, ma cfr. pp. 111 ss. 
sempre fatto ${ }^{53}$. Come si è cercato di mostrare sommariamente, gli esiti sono sempre vari: in sé una tecnica non è né buona né cattiva, e il framework che essa sempre presuppone può essere accettato acriticamente o diventare oggetto di discussione. Su questo crinale si distingueranno, come sempre, le opere interessanti esteticamente e quelle utili come documenti.

Quanto è accaduto negli ultimi trent'anni è dovuto alla nascita di quella che Gianluigi Simonetti chiama "cultura postumanistica" 54 . L'autore di oggi opera "in un ordine culturale mutato, irriducibile alle certezze della tradizione e del buon gusto umanistico" "55. È insomma la "nuova cultura umanistica" sistema che accoglie elementi della cultura tradizionale, ma li pone accanto a oggetti derivati dalla cultura di massa, e al contempo muta il senso di entrambi. Si tratta di un processo ancora in corso: se la letteratura accoglie questa nuova cultura, alcune espressioni di quest'ultima cominciano a essere considerate come fatti estetici, ad esempio il fumetto e le serie televisive. Il successo di una forma $p o p$ di lirica, come quella portata avanti all'interno delle performance dei poetry slam, che oggi sbarca in prima serata ${ }^{57}$, viene pubblicata da editori maggiori e apre i concerti di un cantante irrimediabilmente pop come Dente, è indice di come questa cultura, al di là delle facili tirate apocalittiche, possa rivitalizzare anche un genere socialmente deperito come la lirica. A un costo alto (l'opposizione con la lirica tradizionale, che viene spesso sprezzantemente definita "accademica"), certo. Ma segno di una possibilità di rinascita, attraverso l'ibridazione e la commistione. L'arte non muore, si trasforma.

\section{Carlo Tirinanzi De Medici Università di Trento}

53. Cfr. Bachtin Michail, Estetica e romanzo, Torino, Einaudi, 1979; Rizzante Massimo, Non siamo gli ultimi, Milano, Effigie, 2009, e PArIs Renzo, Romanzi di culto, Roma, Castelvecchi, 1995, p. 28: "Perché spaventarsi oggi se il romanzo è multimediale. È sempre stato spurio (documento, poesia, geografia, lettere, vita, l'extraletterario appunto). Ha perso per questo la sua totalità?”.

54. SimonetTi Gianluigi, La letteratura circostante, cit., p. 47.

55. Ibid., p. 51.

56. Mazzoni Guido, Sulla poesia moderna, Bologna, Il Mulino, 2004, p. 229.

57. I concerti di un cantante pop come Dente vengono aperti da Guido Catalano, un poeta-performer, uno dei primi che si sono sviluppati non tanto in rapporto con la tradizione lirica studiata in accademia, quanto con il sistema mediatico. Uno slammer, Simone Savogin, ha di recente partecipato come poeta a un talent show intitolato Italia's got talent. 
